\title{
Direct laser write process for 3D conductive carbon circuits in polyimide
}

DOI:

10.1039/c7tc01111c

Document Version

Accepted author manuscript

Link to publication record in Manchester Research Explorer

\section{Citation for published version (APA):}

Dorin, B., Parkinson, P., \& Scully, P. (2017). Direct laser write process for 3D conductive carbon circuits in polyimide. Journal of Materials Chemistry C, 5, 4923-4930. https://doi.org/10.1039/c7tc01111c

\section{Published in:}

Journal of Materials Chemistry C

\section{Citing this paper}

Please note that where the full-text provided on Manchester Research Explorer is the Author Accepted Manuscript or Proof version this may differ from the final Published version. If citing, it is advised that you check and use the publisher's definitive version.

\section{General rights}

Copyright and moral rights for the publications made accessible in the Research Explorer are retained by the authors and/or other copyright owners and it is a condition of accessing publications that users recognise and abide by the legal requirements associated with these rights.

\section{Takedown policy}

If you believe that this document breaches copyright please refer to the University of Manchester's Takedown Procedures [http://man.ac.uk/04Y6Bo] or contact uml.scholarlycommunications@manchester.ac.uk providing relevant details, so we can investigate your claim.

\section{OPEN ACCESS}




\title{
Direct laser write process for 3D conductive carbon circuits in polyimide
}

\author{
Bryce Dorin ${ }^{* a, b}$ Patrick Parkinson, ${ }^{a, c}$ and Patricia Scully ${ }^{a, b}$
}

\section{Abstract}

Direct laser writing (DLW) is a versatile materials processing technique often applied to device prototyping. However, a fast and cost effective DLW process for fabricating three-dimensional (3D) conductor-insulator composites has yet to be demonstrated. In this work, polyimide (PI) is established as a viable platform for creating 3D graphitic circuits through ultrafast DLW. Under optimized processing conditions, graphitic material with a resistivity of $6 \Omega \cdot \mathrm{cm}$ was formed in the laser irradiated regions. A thermal and microstructural material model is proposed for the non-linear DLW process and its graphitic products. The process is demonstrated to be an inexpensive and rapid technique for creating electrical contacts to nanoscale components. Future applications of the technique range from nanowire power generation to 3D integrated photonic and electronic devices.

\footnotetext{
0a The Photon Science Institute, The University of Manchester, Oxford Road, Manchester, M13 9PL, United Kingdom. Tel: +44 161275 1000; E-mail: bryce.dorin@manchester.ac.uk

${ }^{0 b}$ School of Chemical Engineering and Analytical Science, The University of Manchester, Oxford Road, Manchester, M13 9PL, United Kingdom.

${ }^{0}$ School of Physics and Astronomy, The University of Manchester, Oxford Road, Manchester, M13 9PL, United Kingdom.

${ }^{0} \dagger$ Electronic Supplementary Information (ESI) available: Beam profiling, Absorption spectroscopy, Pulse energy dependence, Pulse length dependence, Mathematical foundation for the thermal model, Via IV measurements, Electrical turn-on procedure, and Electrical stability measurements.
} 


\section{Introduction}

Over the course of the last 20 years direct laser writing (DLW) has proven itself as a viable technique for three-dimensional (3D) device prototyping in a broad range of transparent materials [1]. The devices that have been demonstrated include photonic components $[2,3]$, laser sources $[4,5]$, and microfluidic channels $[5,6]$. Researchers have also experimented with metal-doped polymers as a material system for DLW sub-surface metallic wires [7-9]. However, using this method the polymer matrix must be washed away before a conductivity can be measured in the DLW metallic structures that remain on the surface. In a limited class of insulating materials it is possible to use DLW to form encapsulated 3D electrically conductive structures. Laser-induced graphitization of diamond was first observed a decade ago [10], and has been exploited to create highly conductive circuits within bulk diamond [11]. Although the material costs for these devices are high, they have applications such as $3 \mathrm{D}$ diamond detectors for particle tracking $[12,13]$.

In this work we establish polyimide (PI) as a new material platform for creating conductive graphitic carbon circuits in 3D. PI is a commercial polymer that is an economical alternative to synthetic diamond. A DLW technique has been developed to induce the formation of functional graphitic material within PI. Several parameters including pulse energy, scan speed, and pulse length have been optimized in the process for peak conductivity and yield. The physical mechanism is confirmed through modelling and microcharacterization of the graphitic carbon product. To demonstrate the potential of this conductor-insulator composite material for rapid device prototyping, electrical contacts are machined through PI to a representative nanotechnology component, namely an encapsulated $\mathrm{Ag}$ nanowire (NW).

PI, also known by its commercial name Kapton, was first developed by DuPont ${ }^{\mathrm{TM}}$. It demonstrates exceptional thermal stability up to $673 \mathrm{~K}$ and good insulating properties $\left(10^{17} \Omega \cdot \mathrm{cm}\right.$ volume resistivity) [14], making it attractive for applications in microelectronics and aerospace. In 1991 Schumann et al. discovered that focusing an ultraviolet (UV) KrF laser onto the surface of PI led to a conductivity increase of 15 orders of magnitude in the irradiated regions [15]. Through Raman spectroscopy it was determined that the irradiated 
sites were largely composed of $\mathrm{C}-\mathrm{C}$ bonds [16]. The work concluded that the $\mathrm{C}=\mathrm{O}, \mathrm{C}-\mathrm{O}$, and $\mathrm{C}-\mathrm{N}$ bonds in the $\mathrm{PI}$ were ruptured during the laser machining process, creating a percolation network of polycrystalline and amorphous carbons. The carbonization of PI is attributed to photothermal effects initiated via laser irradiation. By studying the ablation rate of PI using several laser wavelengths, it was found that the temperature threshold to drive the formation of graphitic carbon is $\sim 850^{\circ} \mathrm{C}$ [17]. In a later study, the gaseous products from PI ablation were captured and measured [18]. The results showed that the ablation products consisted mostly of $\mathrm{CO}$, $\mathrm{HCN}$, and $\mathrm{C}_{2} \mathrm{H}_{2}$.

Infrared (IR) $\mathrm{CO}_{2}$ laser radiation was recently found to be equally capable of producing graphitization on the surface of PI [19]. Further analysis of the irradiated areas using transmission electron microscopy revealed that the laser process liberated carbon monolayers from the PI substrate, creating a porous graphene network on the sample surface. This discovery led to the now widespread use of laser-induced graphene for developing DLW capacitors [20-22] and sensors [23].

Notably, there are no reported studies of sub-surface conductivity increases in the bulk of PI using ultrafast pulses. This is likely due to the very specific beam conditions required to avoid surface damage and still achieve 3D conductive structures. Most work using femtosecond pulsed lasers to machine PI films investigated only laser ablation and surface topology changes [24,25]. Other studies have used femtosecond sources to modify the bulk of PI films [26,27], however no electrical conductivity increases have been reported. To the best of our knowledge, the conversion of bulk PI to conductive graphite using a femtosecond source has not been previously demonstrated in literature. Using the DLW technique outlined here we have been able to fabricate 3D networks of graphitic carbon and create reproducible electrical contacts to nanoscale components (i.e. metallic NWs). This process offers several advantages over current 2D surface patterning techniques, such as electron beam lithography and beam-assisted chemical vapour deposition, commonly used for creating electrical contacts [28]. Using a DLW process, 3D stacks of nanoscale devices and those encapsulated in transparent materials can be contacted with high precision, which would be impossible in a single process otherwise. The reduced number of processing steps 
required and the low cost of materials indicates that this DLW technique may also be attractive to a wide breadth of other electrical prototyping applications.

\section{Experimental}

The PI samples used in this work were cut from Kapton ${ }^{\circledR}$ HN sheets supplied by DuPont ${ }^{\mathrm{TM}}$ with a nominal thickness of $127 \mu \mathrm{m}$. Before laser irradiation the sample surfaces were washed with isopropyl alcohol (IPA) and secured onto glass slides using tape. During DLW the samples were mounted onto an Aerotech ANT130 XYZ stage to achieve positioning with nanometer precision.

The laser source is an amplified high repetition rate Ti:Sapphire system with an output wavelength centred at $800 \mathrm{~nm}$. Seed pulses from a Coherent Mira oscillator are amplified by a Coherent RegA to reach pulse energies on the order of microjoules. For this work the repetition rate of the laser was set to $100 \mathrm{kHz}$. The minimum duration of the output pulses ranged from 150-180 fs, which is monitored using an auto-correlator (assuming a sech ${ }^{2}$ pulse shape). Using the diffraction grating in the RegA the pulses could also be chirped to $1.5 \mathrm{ps}$, used (where stated) for study of the non-linear origin of the breakdown process. The pulse energy of the laser at the sample was controlled using a diffractive optical attenuator. The scan speed of the beam was controlled at the stage from $10-0.002 \mathrm{~mm} / \mathrm{s}$, corresponding to $10-50000$ pulses $/ \mu \mathrm{m}$ at a $100 \mathrm{kHz}$ repetition rates. A linear beam polarization is used during all DLW experiments.

The focusing optic was a $0.45 \mathrm{NA}$ long working distance objective with 20X magnification. Through beam profiling experiments the focal spot size produced by the objective has been calculated to be $2.5 \mu \mathrm{m}$ (see Supplementary Fig. S1). A camera aligned coaxially with the beam was utilized to position the laser spot to specific points on the sample with a precision of $1 \mu \mathrm{m}$. A backlight provides the necessary illumination of the sample.

The Raman spectroscopy measurements we present were carried out on a Renishaw 1000 Raman microscope. The excitation laser source emitted $2.32 \mathrm{~mW}$ of power at a $514 \mathrm{~nm}$ wavelength, which was focused onto the surface of the samples using a $0.55 \mathrm{NA}$ objective. During post-processing the data was smoothed and the fluorescence background from the PI was subtracted. 
The Ag NWs used to demonstrate DLW electrode prototyping were supplied by Sigma-Aldrich (product number: 739448). The nominal dimensions of the NWs were $115 \mathrm{~nm}$ in width and $20-50 \mu \mathrm{m}$ in length, although a small number of wires exceeding $50 \mu \mathrm{m}$ were found. The wires were received dispersed in an IPA solution at a concentration of $0.5 \mathrm{wt} . \%$. The initial solution was diluted to 0.05 wt. $\%$ and spin coated onto a PI film at $5000 \mathrm{rpm}$. Under these conditions isolated NWs were visible on the surface of the PI under 20X magnification. After drying, the surface of the PI samples were covered in a $\sim 1 \mathrm{~mm}$ thick layer of high clarity epoxy (Opti-tec $^{\mathrm{TM}}$ OPT 5012) to encapsulate the Ag NWs.

With the Ag NWs secured and isolated from ambient conditions, graphitic contacts were written to the ends of solitary wires. The initial DLW took place on the surface of the polyimide at the interface with the epoxy. From the DLW contacts, graphitic vias were machined through the bulk of the PI film to the outside surface, providing external electrical links to the fully encapsulated Ag NW. To perform electrical characterization of the DLW structures, silver paint (Alfa Aesar 45661) was applied at the via terminals to form large contacts pads. Gold probes used to contact the pads were connected to a Keithley 487 Picoammeter that acted as a source/measure unit to test the conductivity of the circuits.

\section{Results and Discussion}

From the host of parameters that can be varied in a DLW experiment, optimal conditions were sought that would enable non-linear absorption of the laser beam in the bulk of PI while avoiding ablation at the surface. A critical constraint in any 3D DLW process is the material to be modified must be single-photon transparent to the laser wavelength, which enables absorption to occur solely at the high-intensity laser focus through non-linear processes. The absorption edge of PI was calculated to be $2.3 \mathrm{eV}$ through absorption spectroscopy (see Supplementary Fig. S2). At $800 \mathrm{~nm}$ the Ti:Sapphire laser has a $1.55 \mathrm{eV}$ photon energy, therefore the system satisfies the condition for non-linear photoionization.

To achieve bulk modification, it is important to ensure that the laser fluence at the sample surface is below the ablation threshold of the material. The surface ablation threshold of PI was calculated 

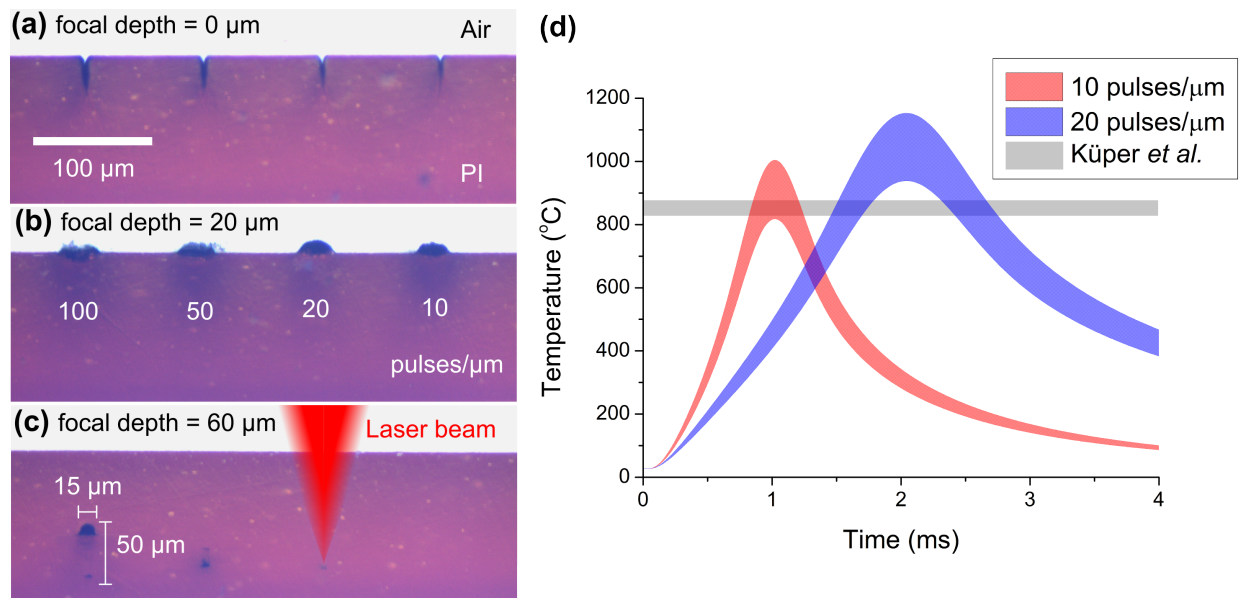

Figure 1: Microscope images (a)-(c) illustrate a cross-sectioned PI film after laser modification at focal depths of 0,20 and $60 \mu \mathrm{m}$, respectively. The materials, scale bar, and scan speeds are common to all three images. The direction of DLW is indicated in (c). For a depth of $60 \mu \mathrm{m}$ the calculated temperature at the focus is plotted as a function of time during DLW and compared with the surface modification threshold reported by Küper et al. in (d) [17]. The area of the calculated curves represents an error of $\pm 10 \%$ in the non-linear absorption coefficient.

by setting the focusing depth (depth of the nominal laser focus below the surface of the sample) to $50 \mu \mathrm{m}$ and scanning the sample transversely at a rate of 10 pulses $/ \mu \mathrm{m}$. Pulse energies of $0.5,1.0,1.5$ and $2.0 \mu \mathrm{J}$ were used. These pulse energies correspond to power intensities of $7 \times 10^{13}, 1.4 \times 10^{14}, 2.1 \times 10^{14}$, and $2.8 \times 10^{14} \mathrm{~W} / \mathrm{cm}^{2}$ at the focus, respectively. A cross-sectional image of the irradiated sample is provided in Supplementary Fig. S3(a). No ablation was measurable for the $0.5 \mu \mathrm{J}$ pulses, however clear damage emerged at 1.0, 1.5 and $2.0 \mu \mathrm{J}$. The ablation threshold for PI was extracted through an analysis of the ablation spot size as a function of laser fluence (see Supplementary Fig. S3(b)) [29]. This threshold was found to be $0.21 \mathrm{~J} / \mathrm{cm}^{2}$, which is equivalent to a pulse energy threshold of $0.95 \mu \mathrm{J}$ for these writing conditions. The result agrees closely with previous work that determined the ablation threshold of PI to be $0.3 \mathrm{~J} / \mathrm{cm}^{2}$ using $800 \mathrm{~nm}$ femtosecond laser irradiation [25]. The reason a lower ablation threshold was observed here may be due to the higher repetition rate of our source $(100 \mathrm{kHz}$ compared to $1 \mathrm{kHz})$, which heats the sample more quickly leading to faster ablation. 
The threshold for bulk modification in PI was determined using several laser scan speeds $(10,20,50$, and 100 pulses $/ \mu \mathrm{m})$ and writing depths $(0,20$, and $60 \mu \mathrm{m})$. The pulse energy was set to $0.5 \mu \mathrm{J}$ to stay well below the ablation threshold previously calculated. Cross-sectional images of the sample after irradiation are provided in Fig. 1(a)-(c). It can be seen that when focused at the surface the laser produces clear ablation spots. When the nominal laser focus is moved $20 \mu \mathrm{m}$ into the bulk of the sample the pulses are still absorbed by the surface of the PI film, which thermally induces the formation of a graphitic material. The graphitic carbon then absorbs any further laser irradiation, preventing modification below the surface. Finally, when the laser is focused $60 \mu \mathrm{m}$ below the surface, the graphitization of bulk PI is detected at $\geq 20$ pulses $/ \mu \mathrm{m}$. No modification is detectable for 10 pulses $/ \mu \mathrm{m}$, which suggests that a temperature threshold must be met before the formation of graphite in the bulk can occur. This is consistent with previous work that proposed a temperature threshold for laser-induced graphitization on the surface of PI [17]. The reason we observe graphite forming at the surface using 10 pulses $/ \mu \mathrm{m}$ in Fig. 1(b) is the thermal diffusivity of the air is much less than that of PI [14], leading to higher peak temperatures at the surface. Scan speeds with 20 and 50 pulses $/ \mu \mathrm{m}$ both produce very faint sub-surface modification regions, which are deeper than $60 \mu \mathrm{m}$ due to spherical aberrations that stretch the focus along its axis. At 100 pulses $/ \mu \mathrm{m}$ a strong modification region $15 \mu \mathrm{m}$ wide becomes clear. One can also see a small secondary modification region $\sim 50 \mu \mathrm{m}$ below the first for 100 pulses $/ \mu \mathrm{m}$, which is again attributed to spherical aberrations [30]. The expected length of focal stretching due to spherical aberrations is calculated to be $50 \mu \mathrm{m}$ (see Supplementary Section S5), which matches these observations. The first modification region exists at the leading edge of the stretched focal region and is formed by the converging paraxial rays of the beam. The second modification region is formed by the aberrated peripheral rays, which avoid absorption in the first modification region and converge deeper within the sample. While this second modification region could also be due to filamentation [31], the large separation between the two regions indicates this is unlikely. It is believed that at higher scan speeds (20 and 50 pulses $/ \mu \mathrm{m})$ there is insufficient energy within the PI to produce modification at the edge of the focal volume, or induce a second modification region. 
This result demonstrates that although PI has a relatively low surface ablation threshold, direct laser writing within the bulk is still possible using low pulse energies and reduced scan speeds.

When the investigation of DLW inside PI was repeated using an $800 \mathrm{~nm}$ continuous-wave beam from the laser oscillator, no modification was observed above or below the surface. In these trials the laser power $(50 \mathrm{~mW})$ and stage scan speeds $(10,20,50$, and 100 pulses $/ \mu \mathrm{m}$ ) were consistent with the work presented in Fig. 1(a)(c). This indicates that short pulses are critical to reach the large peak intensities required for non-linear photoionization. Multiphoton absorption and tunnelling ionization are the two processes responsible for non-linear photoionization [32]. During multiphoton ionization several photons are absorbed simultaneously to create ionization in the sample. For this material system and DLW set-up, 2-photon absorption would achieve sufficient energy for photoionization. In contrast, tunnelling ionization occurs when a large electric field is created at the laser focus, which causes bending of the ionization potential barrier to facilitate tunnelling of the electrons to the excited state. Using the analysis proposed by Keldysh [32] we predict that at a pulse length of $150 \mathrm{fs}$ the initial laser absorption is dominated by the tunnelling ionization process. To further explore the importance of pulse length the experiments outlined in Fig. 1(a)(c) were repeated using pulses chirped to 1.5 ps (see Supplementary Fig. S4). At this longer pulse length the radiation is absorbed in a process dominated by multiphoton photoionization. We observed that 1.5 ps pulses are also capable of producing graphitic carbon in the bulk of PI. Based on this observation we suggest that regardless of the mechanism used to initiate non-linear photoionization, the conversion of bulk PI to graphitic material is ultimately guided by the same photothermal effect.

The results of the scan speed and pulse length dependence experiments indicate there is a temperature threshold that must be reached before graphitization of PI is achieved. Previous work has estimated this temperature threshold to be $852 \pm 26^{\circ} \mathrm{C}$ on the surface [17]. In Fig. 1(d) the temperature of a single point inside of PI is modelled as the laser is scanned over it at a rate of 10 and 20 pulses $/ \mu \mathrm{m}$. This data was obtained using the three-dimensional heat diffusion equation (see Supplementary Section S5 for details). The non-linear absorption of $150 \mathrm{fs}$ long pulses inside the bulk was 
estimated to be $57 \pm 6 \%$ from power transmission experiments (at $0.5 \mu \mathrm{J}$ and 10 pulses $/ \mu \mathrm{m}$ ). This value represents the ratio of power transmitted through PI with the focus centred inside the film to the power transmitted with the focus outside the film. It was assumed that the absorption was spread over the focal volume of the pulse. A focal length of $50 \mu \mathrm{m}$ was determined by calculating the effect of spherical aberrations on the beam, which were confirmed by experimental measurements in Fig. 1(c). The material properties of PI were taken from the material data sheet supplied by DuPont ${ }^{\circledR}$ [14]. We assumed very little heat is able to diffuse to the surface of the PI film, an assumption that is supported by the temperature model itself. From the 20 pulses / $\mu$ m data plot in Fig. $1(d)$ it can be seen that the peak temperature exceeds the threshold calculated by Küper et al. within error [17]. This result supports our experimental observations that a scan speed of 20 pulses $/ \mu$ m produces temperatures inside the PI above the graphitization threshold. When 10 pulses $/ \mu \mathrm{m}$ is simulated, the peak temperature overlaps with the threshold within error. This result does not allow us to confirm or deny our experimental observation that 10 pulses $/ \mu \mathrm{m}$ is below the graphitization threshold of PI. However, it does indicate that at 10 pulses $/ \mu \mathrm{m}$ the temperature inside the PI is close to the threshold. Therefore, if modification is achieved it may be too weak to resolve through our microscopy set-up. Together these results indicate that the surface graphitization temperature threshold may also apply to graphitization in the bulk.

Experiments were carried out to create conductive graphitic structures through the bulk of PI films in the form of vias using 3D DLW. The laser pulse energy was again set to $0.5 \mu \mathrm{J}$ while varying the stage scan speed from $1000-50000$ pulses $/ \mu \mathrm{m}$ to find the optimal conditions for DLW. The writing direction was always perpendicular to beam polarization. During experiments the focus of the laser was traced from the back of the samples, through the bulk, to the front in order to avoid surface ablation. Five vias were fabricated at each scan speed. It was found that in all tests the vias were initially very resistive at low voltages, but when higher voltages were applied some devices displayed a turn-on behaviour during a 0-500-0 V scan. In Fig. 2(a) the probability a via will turn-on and the average resistance of those that did are plotted as a function of scan speed. The resistance was measured from a $-2-2$ V IV scan where all devices be- 

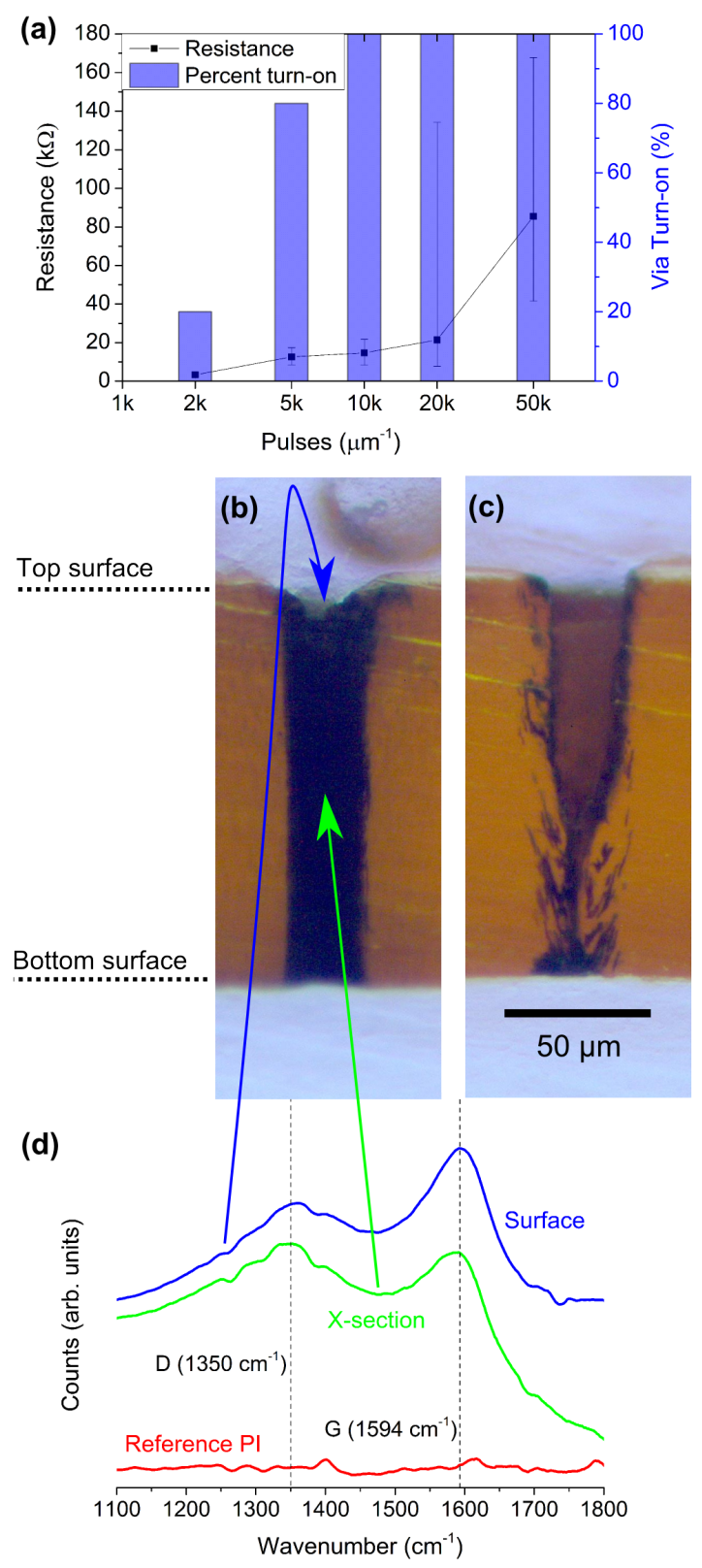

Figure 2: The conductivity and turn-on probabilities for vias are plotted as a function of scan speed in (a). Error is defined by the maximum and minimum resistance values measured in each set of vias. Cross-sections of DLW vias through PI written with 10000 pulses/ $\mu \mathrm{m}$ back-to-front and front-to-back are imaged optically in (b) and (c), respectively. For the back-to-front via a Raman spectrum from the surface and bulk is plotted in (d). 
haved ohmically above $1 \mathrm{~V}$ (see Supplementary Fig. S5). The error for the resistance values is taken from the maximum and minimum measured resistances of the 5 devices tested. It can be seen from this plot that 10000 pulses / $\mu \mathrm{m}$ was sufficient to generate turn-on in all vias fabricated with an average resistance of $14_{-6}^{+7} \mathrm{k} \Omega$. Although reducing the scan speed still creates devices that consistently turnon, the average resistance of the vias is also increased. Vias were also fabricated using 1.5 ps pulses and 10000 pulses $/ \mu \mathrm{m}$, however the average resistance of the vias was marginally higher $\left(16_{-8}^{+9} \mathrm{k} \Omega\right)$ and only $80 \%$ of the devices turned-on.

Supplementary Fig. S6 illustrates the via turn-on behaviour. The current measured through the via quickly ( $i 1 \mathrm{~s})$ spikes as the voltage rises during turn-on, indicating a high conductivity state has formed. This behaviour is similar to resistive switching that has been seen in carbon devices before [33]. The proposed switching mechanism in these devices is Joule heating within the nanocrystalline microstrucuture of the DLW region, which produces a conductive filament through annealing $[34,35]$. The physical effect of the annealing is believed to be clustering and alignment of the $\mathrm{sp}^{2}$ hybridized sites within the $\mathrm{sp}^{3}$ matrix of the amorphous carbon along the direction of the applied bias. In previous studies groups have also been able to turn-off the devices and achieve bi-polar switching using carbon. This was done through the application of 0.1 ns voltage pulses that heat-quench the carbon, breaking the conductive pathways [36]. This turn-off effect was not desired in our application, therefore the voltage is always reduced slowly in the device to avoid quenching the conductivity. The stability of the via following device turn-on was measured over the course of 40 hours (see Supplementary Fig. S7). During this time the current dropped by only $0.06 \%$ /hour, and was not affected by the absence of any applied bias for one hour.

This same microstructural model for the graphitic material produced through DLW can be applied to understand the rising resistances seen at scan speeds slower than 10000 pulses $/ \mu \mathrm{m}$. Slower scan speeds will create higher temperatures inside the PI, ultimately leading to larger quenching effects once the laser is removed from the film. These quenching effects may create extensive breaks within the conductive layers of graphite, similar to turn-off in carbon-based bipolar switches. For this reason 10000 pulses $/ \mu \mathrm{m}$ was chosen as the ideal scan speed in this process. As a demonstration that 3D DLW is 
essential to this technique, 5 vias were fabricated at 10000 pulses $/ \mu \mathrm{m}$ with the laser scanning from the front to the back of the sample. In this case no conductive devices were formed, which is likely because the ablation/modification of the PI surface hinders the formation of graphite within the bulk.

The cross-section of a via written back-to-front at 10000 pulses $/ \mu \mathrm{m}$ (which are measured in Fig. 2(a)) is presented in Fig. 2(b). The cross-section of a second via written front-to-back at 10000 pulses $/ \mu \mathrm{m}$ is provided in Fig. 2(c). One can see that the former of the two has a very consistent cylindrical shape through the bulk of the film. Only a small amount of ablation is visible on the front surface of the via. Taking the width of the via $(w)$ to be $\sim 25 \mu \mathrm{m}$ and the average resistance $(R)$ to be $14 \mathrm{k} \Omega$ (from Supplementary Fig. S5), the volume resistivity $(\rho)$ of the modified area can be calculated using $\rho=R \pi w^{2} / 4 t$, where $t$ is the thickness of the film $(127 \mu \mathrm{m})$. Using this expression the resistivity is calculated to be approximately $6 \Omega \cdot \mathrm{cm}$, which is 16 orders of magnitude less than virgin PI and 2-3 orders of magnitude greater than pure graphite. This resistivity is also 2-3 orders of magnitude larger than for DLW wires produced on the surface of PI using the same laser source, the reason for which is unclear. In image Fig. 2(c) where the wire was written frontto-back, the shape of the modification is clearly very distict from the via written back-to-front. Instead of a graphitic wire we see a large ablation area leading from the front surface to the middle of the film. There is no complete conductive pathway visible in this image, which is consistent with its insulating properties.

Raman spectroscopy was performed in the bulk of the via and on the surface (Fig. $2(\mathrm{~d})$ ), where the $\mathrm{D}$ and $\mathrm{G}$ peaks of graphite are clearly visible. From the location of the $G$ peak $\left(\sim 1600 \mathrm{~cm}^{-1}\right)$ we can infer that clustering of the conductive $\mathrm{sp}^{2}$ graphite phase is present, indicating a nanocrystalline microstructure [37]. The presence and size of the $\mathrm{D}$ peak also supports this conclusion. This microstructural model is in agreement with the observation of resistive switching behaviour in the vias.

Based on these results we propose the laser-induced conversion of bulk PI to graphite develops as follows. Non-linear photonionization processes lead to absorption of the ultrafast beam below the surface of PI. If the scan speed of the process is sufficiently slow, heat will build up at the laser focus to exceed the thermal thresh- 

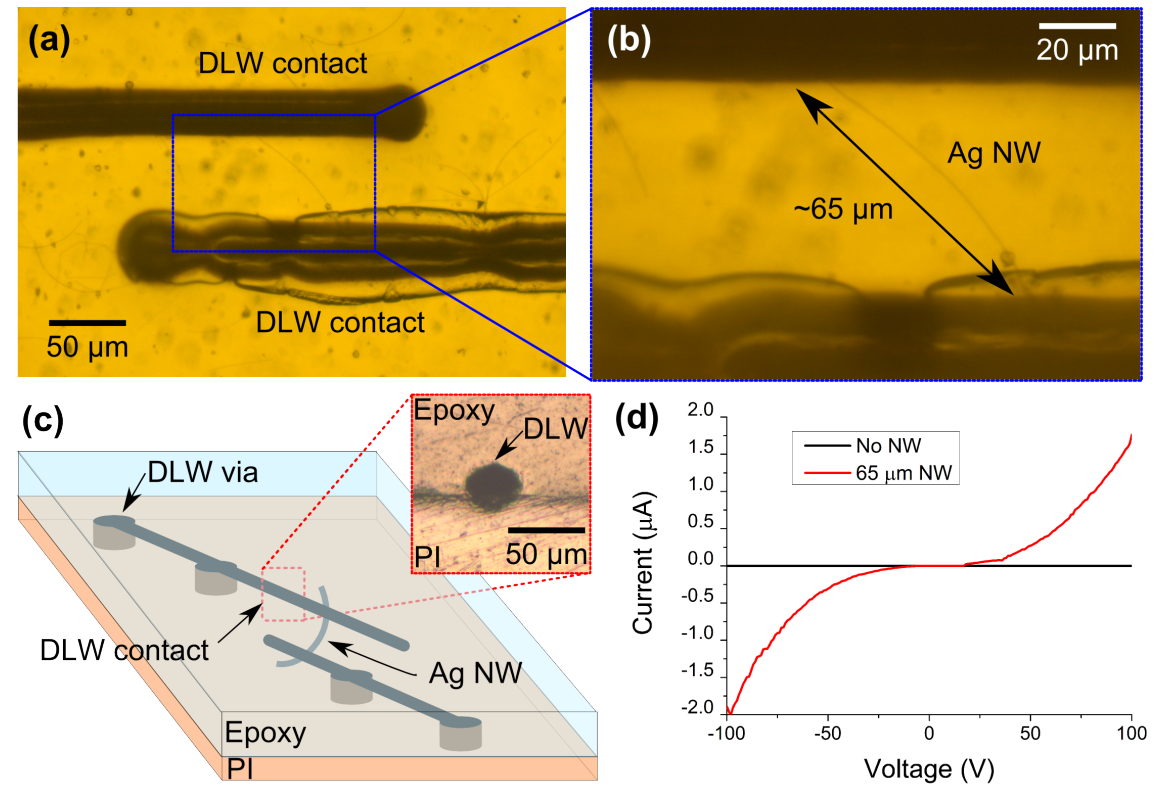

Figure 3: DLW contacts to a silver NW are imaged through the epoxy encapsulate under 20X and 50X magnification in images (a) and (b), respectively. The schematic in (c) illustrates the complete DLW circuit to the NW. The inset is a cross-sectional image of a DLW contact. An IV plot for the NW circuit is provided in $(\mathrm{d})$, with the IV data for an identical circuit without a NW added for reference.

old for graphitization $\left(\sim 850^{\circ} \mathrm{C}\right)$. At this temperature the $\mathrm{C}=\mathrm{O}$, $\mathrm{C}-\mathrm{O}$, and $\mathrm{C}-\mathrm{N}$ bonds in the $\mathrm{PI}$ will rupture and be replaced by $\mathrm{C}-\mathrm{C}$ bonds [16]. The possible release of $\mathrm{CO}, \mathrm{HCN}$, and $\mathrm{C}_{2} \mathrm{H}_{2}$ gases into the bulk of the PI during the process is the subject of future work [18]. The laser-modified region then contains isolated graphitic nanocrystals in an amorphous carbon network. Joule heating along the DLW path selectively anneals a filament through the graphitic carbons. The final product is a conductive pathway through percolative clusters of aligned nanocrystals.

We demonstrate the new capabilities that this technique provides by applying our 3D DLW process to the electrical packaging of exemplary nanostructures. NWs are notoriously difficult to drive electrically as their small size requires electrical contacts to be fabricated with high precision. Their small size also makes them difficult to position laterally across pre-existing contacts on a substrate, although techniques such as fluidic alignment [38] and dielec- 
trophoresis $[39,40]$ have been demonstrated. Ultimately, forming electrical contacts to single randomly deposited NWs usually requires electron-beam lithography or beam-assisted chemical vapour deposition [28]. The PI/epoxy packaging system used here to encapsulate the NWs is appealing for several reasons. The PI itself is a chemically/thermally stable and flexible polymer that has been used repeatedly in the past as a substrate for NW devices [41-45]. The epoxy encapsulate serves to confine the NWs and protect them from ambient conditions, which may eliminate the need for controlled atmospheres during fabrication and testing in some applications.

An encapsulated Ag NW with DLW graphitic electrodes is presented in Fig. 3(a) and (b). The wires were fabricated at the interface between the PI and epoxy using a $1.0 \mu \mathrm{J}$ pulse energy and 20 pulses $/ \mu \mathrm{m}$. The pulse energy was chosen to be above the surface ablation threshold of PI. The path of the laser was directed through the epoxy encapsulate for this process. We found that the direction of writing in relation to beam polarization had no significant effect on conductivity, although recent work has indicated polarization can play a role in photothermal processes and further work is required to confirm this [46]. From the surface wires, vias were fabricated through the PI film to form external contacts. This process used a $0.5 \mu \mathrm{J}$ pulse energy and 10000 pulses $/ \mu \mathrm{m}$, as these parameters were deemed to be optimal in previous experiments. To perform this the sample was flipped so the beam was now incident on the PI film. A schematic detailing the final DLW circuit is provided in Fig. 3(c). Via-wire contact was made by using vias in pairs, which could be turned-on in isolation to avoid applying large voltages across the NW. The inset of Fig. 3(c) is a cross-sectional image of a DLW wire formed on the surface of encapsulated PI. Here, one can see the graphitic carbon formed through DLW has pushed into the epoxy layer, similar to Fig. 1(b). It is believed that the heat generated during the DLW process softens the epoxy to allow for this effect. The current measured through the NW after all vias had been turned on is shown in the IV plot in Fig. 3(d). For reference, the same DLW circuit was fabricated without a NW in the gap between surface contacts. No conductance was measurable through this device, indicating that the current measured in Fig. 3(d) is only passing through the NW and not conducting through the PI or epoxy films. The electrical response of the circuit is highly non-linear, with a resis- 
tance of $50 \mathrm{M} \Omega$ at $100 \mathrm{~V}$. When the device was pushed to $145 \mathrm{~V}$ the contact to the NW failed, although no damage was visible through microscopy. Independent resistance measurements of vias, wires at the interface of PI and epoxy, and the junctions between the two indicate that the DLW components of the circuit account for a resistance of $66_{-32}^{+239} \mathrm{k} \Omega$. The Ag NWs themselves have a negligible resistance, therefore the majority of the resistance occurs at the DLW junction with the NW, which was not optimized here.

\section{Conclusions}

This work demonstrates a one step DLW process for fabricating 3D conductor-insulator composite materials using commercial polymer films. The effects of pulse energy, pulse length, and scan speed have been studied in-depth to determine the optimal parameters for creating 3D electrical conductors in PI. Thermal modelling of the process confirmed that the temperature threshold for laser-induced sub-surface graphitization is $\sim 850^{\circ} \mathrm{C}$. Graphitic carbon vias with a resistivity of $6 \Omega \cdot \mathrm{cm}$ were formed through the bulk of PI films. Our analysis of these vias concludes that the DLW regions are composed of nanocrystalline $\mathrm{sp}^{2}$ hybridized carbons within the $\mathrm{sp}^{3}$ hybridized matrix. By selectively annealing the irradiated regions using Joule heating the $\mathrm{sp}^{2}$ sites cluster to form a conductive filament. This microstructural material model is consistent with previous work [16, 35] and the analysis of our Raman spectra.

This novel 3D graphite-PI material system shows promise in the field of micro/nano-electronics packaging. As a proof of principle, the optimized DLW methodology was applied to fabricate electrical contacts across an encapsulated Ag NW. Looking forward, the same process could be used to realize electrically driven NW power generators [41], switches [42], heterojunctions [43], transistors [44], and sensors [45] on flexible PI substrates. Our results demonstrate the DLW process is also possible using a picosecond laser source, which dramatically reduces the cost of applying it in a commercial setting. It is feasible that the same laser source could be used to characterize the performance of functional NWs $[47,48]$ before contacts are written to the best performing devices. Finally, the versatility of DLW suggests that fabricating electrical circuits and photonic components could be achieved using a single methodology. Coating 
materials that support DLW optical waveguides, such as glass $[1,2]$ and polymers [49], with PI films may prove to be a viable platform for integrating optics and electronics.

\section{Acknowledgements}

Research funding was provided through the President's Doctoral Scholarship awarded to BD from The University of Manchester. PP acknowledges funding from the Royal Society (RG140411). PS ac-

knowledges funding from The University of Manchester School of Chemical Engineering and Analytical Science Pump Prime Fund.

\section{References}

[1] R. R. Gattass and E. Mazur, "Femtosecond laser micromachining in transparent materials," Nature Photonics, vol. 2, pp. 219 $225,2008$.

[2] K. Miura, J. Qiu, H. Inouye, T. Mitsuyu, and K. Hirao, "Photowritten optical waveguides in various glasses with ultrashort pulse laser," Applied Physics Letters, vol. 71, p. 3329, 1997.

[3] G. Della Valle, R. Osellame, and P. Laporta, "Micromachining of photonic devices by femtosecond laser pulses," Journal of Optics A, vol. 11, p. 013001, jan 2009.

[4] G. A. Torchia, A. Rodenas, A. Benayas, E. Cantelar, L. Roso, and D. Jaque, "Highly efficient laser action in femtosecondwritten Nd:yttrium aluminum garnet ceramic waveguides," $A p$ plied Physics Letters, vol. 92, p. 111103, 2008.

[5] D. Choudhury, J. R. Macdonald, and A. K. Kar, "Ultrafast laser inscription: perspectives on future integrated applications," Laser and Photonics Reviews, pp. 1-20, may 2014.

[6] Y. Bellouard, A. Said, M. Dugan, and P. Bado, "Fabrication of high-aspect ratio, micro-fluidic channels and tunnels using femtosecond laser pulses and chemical etching," Optics Express, vol. 12, no. 10, p. 2120, 2004. 
[7] C. N. Lafratta, D. Lim, K. O. Malley, T. Baldacchini, and J. T. Fourkas, "Direct Laser Patterning of Conductive Wires on Three-Dimensional Polymeric Microstructures," Chemistry of Materials, vol. 23, no. 5, pp. 2038-2042, 2006.

[8] S. Maruo and T. Saeki, "Femtosecond laser direct writing of metallic microstructures by photoreduction of silver nitrate in a polymer matrix," Optics Express, vol. 16, no. 2, pp. 11741179, 2008.

[9] R. Nakamura, K. Kinashi, W. Sakai, and N. Tsutsumi, "Fabrication of the silver structure through two-photon excitation by femtosecond laser," Chemical Physics Letters, vol. 610-611, pp. 241-245, 2014.

[10] T. V. Kononenko, M. Meier, M. S. Komlenok, S. M. Pimenov, V. Romano, V. P. Pashinin, and V. I. Konov, "Microstructuring of diamond bulk by IR femtosecond laser pulses," Applied Physics A, vol. 90, pp. 645-651, 2008.

[11] B. Sun, P. S. Salter, and M. J. Booth, "High conductivity microwires in diamond following arbitrary paths," Applied Physics Letters, vol. 105, p. 231105, 2014.

[12] S. Lagomarsino, M. Bellini, C. Corsi, S. Fanetti, F. Gorelli, I. Liontos, G. Parrini, M. Santoro, and S. Sciortino, "Electrical and Raman-imaging characterization of laser-made electrodes for 3D diamond detectors," Diamond and Related Materials, vol. 43, pp. 23-28, 2014.

[13] F. Bachmair, L. Bäni, P. Bergonzo, B. Caylar, G. Forcolin, I. Haughton, D. Hits, H. Kagan, R. Kass, L. Li, A. Oh, S. Phan, M. Pomorski, D. S. Smith, V. Tyzhnevyi, R. Wallny, and D. Whitehead, "A 3D diamond detector for particle tracking," Nuclear Instruments and Methods in Physics Research, Section $A$, vol. 786, pp. 97-104, 2015.

[14] DuPont, "Technical Data Sheet, Kapton @ HN," 2011.

[15] M. Schumann, R. Sauerbrey, and M. C. Smayling, "Permanent increase of the electrical conductivity of polymers induced by ultraviolet laser radiation," Applied Physics Letters, vol. 58, p. $428,1991$. 
[16] X. Gu, "Raman spectroscopy and the effects of ultraviolet irradiation on polyimide film," Applied Physics Letters, vol. 62, pp. 1568-1570, 1993.

[17] S. Küper, J. Brannon, and K. Brannon, "Threshold behavior in polyimide photoablation: Single-shot rate measurements and surface-temperature modeling," Applied Physics A, vol. 56, pp. 43-50, 1993.

[18] R. Srinivasan, R. R. Hall, W. D. Loehle, W. D. Wilson, and D. C. Allbee, "Chemical transformations of the polyimide Kapton brought about by ultraviolet laser radiation," Journal of Applied Physics, vol. 78, pp. 4881-4887, 1995.

[19] J. Lin, Z. Peng, Y. Liu, F. Ruiz-Zepeda, R. Ye, E. L. G. Samuel, M. J. Yacaman, B. I. Yakobson, and J. M. Tour, "Laser-induced porous graphene films from commercial polymers," Nature Communications, vol. 5, no. 5714, pp. 1-8, 2014.

[20] Z. Peng, J. Lin, R. Ye, E. L. G. Samuel, and J. M. Tour, "Flexible and Stackable Laser-Induced Graphene Supercapacitors.," ACS applied materials \& interfaces, vol. 7, pp. 3414-9, 2015.

[21] Z. Peng, R. Ye, J. A. Mann, D. Zakhidov, Y. Li, P. R. Smalley, J. Lin, and J. M. Tour, "Flexible Boron-Doped Laser Induced Graphene Microsupercapacitors," ACS Nano, vol. 9, no. 6, pp. 5868-5875, 2015.

[22] J. B. In, B. Hsia, J. H. Yoo, S. Hyun, C. Carraro, R. Maboudian, and C. P. Grigoropoulos, "Facile fabrication of flexible all solidstate micro-supercapacitor by direct laser writing of porous carbon in polyimide," Carbon, vol. 83, pp. 144-151, 2015.

[23] S. Luo, P. T. Hoang, and T. Liu, "Direct laser writing for creating porous graphitic structures and their use for flexible and highly sensitive sensor and sensor arrays," Carbon, vol. 96, pp. 522-531, 2016.

[24] H. Kumagai, K. Midorikawa, K. Toyoda, S. Nakamura, T. Okamoto, and M. Obara, "Ablation of polymer films by a femtosecond high-peak-power Ti:sapphire laser at $798 \mathrm{~nm}$," Applied Physics Letters, vol. 65, pp. 1850-1852, 1994. 
[25] B. S. Haq, H. U. Khan, K. Alam, M. Mateenullah, S. Attaullah, and I. Zari, "Femtosecond pulsed laser ablation of polyimide at oblique angles for medical applications," Applied Optics, vol. 54, no. 24, pp. 7413-7418, 2015.

[26] J. Morikawa, A. Orie, T. Hashimoto, and S. Juodkazis, "Thermal diffusivity in femtosecond-laser-structured micro-volumes of polymers," Applied Physics A, vol. 98, pp. 551-556, 2010.

[27] S. Wang, R. Li, C. Zheng, C. Cheng, Y. Yu, S. Bai, G. Feng, and $\mathrm{A} . \mathrm{Hu}$, "Laser direct writing and photonic manufacturing of microball lens for wide-angle imaging, energy devices, and sensors on flexible substrates," in 17th International Symposium on Laser Precision Microfabrication Laser, 2016.

[28] T. Hanrath and B. A. Korgel, "Germanium nanowire transistors: a comparison of electrical contacts patterned by electron beam lithography and beam-assisted chemical vapour deposition," Proceedings of the Institution of Mechanical Engineers, Part N, vol. 218, pp. 25-34, 2005.

[29] M. J. Liu, "Simple technique for measurements of pulsed Gaussian-beam spot sizes," Opt. Lett., vol. 7, no. 5, pp. 196198, 1982.

[30] S. Hell, G. Reiner, C. Cremer, and E. H. K. Stelzer, "Abberations in confocal fluporescence microscopy induced by mismatches in refractive index," Journal of Microscopy, vol. 169, no. 3, pp. 391-405, 1993.

[31] K. Yamada, W. Watanabe, T. Toma, K. Itoh, and J. Nishii, "In situ observation of photoinduced refractive-index changes in filaments formed in glasses by femtosecond laser pulses," Optics letters, vol. 26, pp. 19-21, jan 2001.

[32] L. V. Keldysh, "Ionization in the field of a strong electromagnetic wave," Soviet Physics JETP, vol. 20, no. 5, pp. 1307-1314, 1965 .

[33] C. A. Santini, A. Sebastian, C. Marchiori, V. P. Jonnalagadda, L. Dellmann, W. W. Koelmans, M. D. Rossell, C. P. Rossel, and E. Eleftheriou, "Oxygenated amorphous carbon for resistive 
memory applications," Nature Communications, vol. 6, p. 8600, 2015.

[34] A. Sebastian, A. Pauza, C. Rossel, R. M. Shelby, A. F. Rodríguez, H. Pozidis, and E. Eleftheriou, "Resistance switching at the nanometre scale in amorphous carbon," New Journal of Physics, vol. 13, p. 013020, 2011.

[35] J. Y. Huang, S. Chen, Z. F. Ren, G. Chen, and M. S. Dresselhaus, "Real-time observation of tubule formation from amorphous carbon nanowires under high-bias Joule heating," Nano Letters, vol. 6, no. 8, pp. 1699-1705, 2006.

[36] Y. He, J. Zhang, X. Guan, L. Zhao, Y. Wang, H. Qian, and $\mathrm{Z}$. Yu, "Molecular dynamics study of the switching mechanism of carbon-based resistive memory," IEEE Transactions on Electron Devices, vol. 57, no. 12, pp. 3434-3441, 2010.

[37] A. Ferrari and J. Robertson, "Interpretation of Raman spectra of disordered and amorphous carbon," Physical Review B, vol. 61, no. 20, pp. 14095-14107, 2000.

[38] Y. Huang, X. Duan, Q. Wei, and C. M. Lieber, "Directed Assembly of One-Dimensional Nanostructures into Functional Networks," Science, vol. 291, pp. 630-633, 2001.

[39] S. Raychaudhuri, S. A. Dayeh, D. Wang, and E. T. Yu, "Precise semiconductor nanowire placement through dielectrophoresis," Nano Letters, vol. 9, no. 6, pp. 2260-2266, 2009.

[40] E. M. Freer, O. Grachev, D. P. Stumbo, X. Duan, S. Martin, and D. P. Stumbo, "High-yield self-limiting single-nanowire assembly with dielectrophoresis," Nature nanotechnology, vol. 5, pp. 525-530, 2010.

[41] R. Yang, Y. Qin, L. Dai, and Z. L. Wang, "Power generation with laterally packaged piezoelectric fine wires," Nature nanotechnology, vol. 4, pp. 34-39, 2009.

[42] Y. Dong, G. Yu, M. C. McAlpine, W. Lu, and C. M. Lieber, "Si/a-Si core/shell nanowires as nonvolatile crossbar switches," Nano Letters, vol. 8, no. 2, pp. 386-391, 2008. 
[43] J. Park, Y. Kim, G. T. Kim, and J. S. Ha, "Facile fabrication of SWCNT/SnO2 nanowire heterojunction devices on flexible polyimide substrate," Advanced Functional Materials, vol. 21, pp. 4159-4165, 2011.

[44] B. P. Timko, T. Cohen-Karni, G. Yu, Q. Qing, B. Tian, and C. M. Lieber, "Electrical recording from hearts with flexible nanowire device arrays," Nano Letters, vol. 9, no. 2, pp. 914918,2009 .

[45] K. Takei, T. Takahashi, J. C. Ho, H. Ko, A. G. Gillies, P. W. Leu, R. S. Fearing, and A. Javey, "Nanowire active-matrix circuitry for low-voltage macroscale artificial skin.," Nature materials, vol. 9, no. 10, pp. 821-6, 2010.

[46] S. Rekstyte, T. Jonavicius, D. Gailevicius, M. Malinauskas, V. Mizeikis, E. G. Gamaly, and S. Juodkazis, "Nanoscale Precision of 3D Polymerization via Polarization Control," Advanced Optical Materials, vol. 4, no. 8, pp. 1209-1214, 2016.

[47] P. Parkinson, Y. H. Lee, L. Fu, S. Breuer, H. H. Tan, and C. Jagadish, "Three-dimensional in situ photocurrent mapping for nanowire photovoltaics," Nano Letters, vol. 13, pp. 14051409, 2013.

[48] K. Peng, P. Parkinson, L. Fu, Q. Gao, N. Jiang, Y.-N. Guo, F. Wang, H. Joyce, J. Boland, M. Johnston, H. Tan, and C. Jagadish, "Single Nanowire Terahertz Detectors," Nano Letters, vol. 15, pp. 206-210, 2015.

[49] A. Baum, P. J. Scully, M. Basanta, C. L. Paul Thomas, P. R. Fielden, N. J. Goddard, W. Perrie, and P. R. Chalker, "Photochemistry of refractive index structures in poly(methyl methacrylate) by femtosecond laser irradiation," Optics Letters, vol. 32, no. 2, pp. 190-192, 2007. 